\title{
Fetal alcohol spectrum disorder: a guideline for diagnosis across the lifespan
}

\author{
Jocelynn L. Cook PhD, Courtney R. Green PhD, Christine M. Lilley PhD, Sally M. Anderson PhD, \\ Mary Ellen Baldwin, Albert E. Chudley MD, Julianne L. Conry PhD, Nicole LeBlanc MD, \\ Christine A. Loock MD, Jan Lutke, Bernadene F. Mallon MSW, Audrey A. McFarlane MBA, \\ Valerie K. Temple PhD, Ted Rosales MD; for the Canada Fetal Alcohol Spectrum Disorder \\ Research Network
}

CMAJ Podcasts: author interview at https://soundcloud.com/cmajpodcasts/141593-guide

See also www.cmaj.ca/lookup/doi/10.1503/cmaj.151425

$\mathrm{T}$ he consequences of prenatal alcohol exposure were first described more than 40 years ago. ${ }^{1,2}$ The term "fetal alcohol syndrome" (FAS) was first used to describe the cluster of birth defects due to prenatal alcohol exposure (including growth restriction, craniofacial abnormalities and intellectual disabilities) with lifetime consequences. ${ }^{2}$ The term "fetal alcohol spectrum disorder" (FASD) has since been adopted to describe a broader spectrum of presentations and disabilities resulting from alcohol exposure in utero. The prevalence has been estimated at 1 in 100 people, which translates to more than 330000 affected individuals in Canada. ${ }^{3}$

The development of clinical capacity for FASD diagnosis remains difficult, ${ }^{4}$ because the diagnosis requires a medical evaluation and neurodevelopmental assessment conducted by a multidisciplinary team. In 2005, an international, collaborative, evidence-based guideline for diagnoses related to prenatal alcohol exposure was published. ${ }^{5}$ Since then, the field has evolved, and additional evidence, expertise and experience have emerged to suggest that a revision was required to improve both diagnoses and outcomes. The literature has also shown that impairments in behaviour and function associated with FASD have been detected from exposure to binge drinking, even infrequently or early in pregnancy, which underscores the importance of pre-pregnancy counselling. Specific research involving infants, young children and adults with FASD, as well as further insight into the neurodevelopmental dysfunction and nomenclature, prompted the update and revision process. A literature review and broad consultation process was undertaken to revise the 2005 guideline for diagnosing FASD. ${ }^{5}$

\section{Scope}

Recommendations are focused on the diagnostic process and are geared toward members of multidisciplinary diagnostic teams in Canada, who have received the required expertise and experience through specialized training. Although primary health care providers, who provide antenatal care and counsel individuals considering pregnancy, may also benefit from these recommendations, the diagnostic process should not be performed in isolation; multidisciplinary input is required.

\section{Methods}

\section{Guideline steering committee}

A 14-member steering committee was formed in September 2012. Members were selected by the Canada Fetal Alcohol Spectrum Disorder Research Network based on previous involvement with the 2005 diagnostic guideline, expertise in FASD and expertise in areas requiring specific attention (e.g., diagnostic guidelines for infants and young children, and adults; nomenclature; and the neurodevelopmental assessment criteria). The committee consisted of four psychologists, three researchers, three pediatricians, one social worker, one clinical geneticist, one FASD clinic coordinator and one parent of individuals living with FASD.
Competing interests: None declared.

This article has been peer reviewed.

Correspondence to: Jocelynn Cook, jcook@sogc.com

CMAJ 2016. DOI:10.1503 /cmaj.141593

\section{KEY POINTS}

- Fetal alcohol spectrum disorder (FASD) is a diagnostic term describing the constellation of effects that result from prenatal alcohol exposure.

- Making a diagnosis of FASD requires a multidisciplinary team and involves a complex physical and neurodevelopmental assessment.

- Diagnosis of FASD is critical to improve outcomes for affected individuals and families, and to inform pre-pregnancy counselling to prevent future cases. 


\section{Guideline development}

The guideline was developed according to the Appraisal of Guidelines, Research and Evaluation (AGREE II) framework, ${ }^{6}$ which provides a rigorous, evidence-based approach. The steering committee identified the scope of the guidelines and developed the key questions. These tasks were accomplished during the first meetings, after which the scope and questions were further refined and approved by consensus. The literature review was then conducted by two committee members (J.L.C. and C.R.G.) using the following combinations of MeSH (medical subject heading) search terms to address the key questions: "fetal alcohol," "diagnosis*," "infant*," "prenatal alcohol" and "adult." Relevant reports published from 2005 to September 2014 were identified from the following databases: PubMed, PsychLIT, Medscape, MEDLINE, the Canadian Institutes of Health Research funding database and the Cochrane Library.

To supplement the literature review, the steering committee gleaned input from diagnostic centres across Canada; 35 clinics completed an online survey to identify limitations in the current diagnostic process. The survey was developed and customized by the steering committee to ensure that the necessary information was collected to address the key questions identified for this project. As well, national and international expert consultations were conducted via six inperson focus groups before the recommendations were developed. Three of the focus groups comprised all members of the steering committee, and two smaller focus groups consisted of two committee members and an international FASD researcher and clinician.

\section{Box 1: Grading of recommendations ${ }^{7-9}$}

\section{Strength of the recommendation}

- Strong: Highly confident of the balance between desirable and undesirable consequences (i.e., desirable consequences outweigh the undesirable consequences; or undesirable consequences outweigh the desirable consequences).

- Weak*: Less confident of the balance between desirable and undesirable consequences.

\section{Quality level of evidence}

- High: We are very confident that the true effect lies close to that of the estimate of the effect.

- Moderate: We are moderately confident in the effect estimate. The true effect is likely to be close to the estimate of the effect, but there is a possibility that it is substantially different.

- Low: Our confidence in the effect estimate is limited. The true effect may be substantially different from the estimate of the effect.

- Very low: We have very little confidence in the effect estimate: The true effect is likely to be substantially different from the estimate of effect

*Weak recommendations should not be misinterpreted as weak evidence or uncertainty of the recommendation.
Finally, a two-day international focus group consisting of 50 participants (including a facilitator and administrative assistant) was held. Participants were invited to provide input and advice for recommendations based on their FASD expertise as it related to diagnosis (specifically infants, young children and adults with FASD, and the neurodevelopmental assessment). The participants included 19 psychologists, 11 pediatricians, 5 researchers, 3 speech and language pathologists, 3 occupational therapists, 2 psychiatrists, 1 clinical geneticist, 1 policy director, 1 parent, 1 social worker and 1 clinic coordinator.

When the literature review activities were completed, summaries and reports were prepared by two experienced researchers (J.L.C. and C.R.G.). These materials were circulated to all members of the steering committee for their review and to inform development of the recommendations. Once the recommendations were drafted by the steering committee, they were appraised independently by the same two researchers using the Grading of Recommendations Assessment, Development and Evaluation (GRADE) approach ${ }^{7-9}$ (Box 1). The recommendations and rankings were reviewed three times by committee members before a penultimate version was circulated to external national and international experts for further feedback. All comments from these reviews were collated (by C.R.G) and circulated back to the steering committee for consideration and further discussion (at teleconference meetings) until each recommendation reflected the consensus of the committee. When there were discrepancies, recommendations were further refined to ensure there was unanimous endorsement. More information on the development of the guideline can be found in Appendix 1 (available at www.cmaj.ca/ lookup/suppl/doi:10.1503/cmaj.141593/-/DC1).

\section{Recommendations}

The guideline provides recommendations on the screening, referral and support for pregnant or postpartum women and for individuals at risk of FASD; the medical assessment, including family history, maternal alcohol history, physical examination and differential diagnosis; the sentinel facial features; the neurodevelopmental assessment; the nomenclature and diagnostic criteria; and the diagnostic team and special considerations in the neurodevelopmental assessment of infants and young children.

The recommendations are outlined in Table 1. Details of the evidence underpinning the recommendations can be found in the full- 
Table 1 (part 1 of 2): Recommendations for the diagnosis of fetal alcohol spectrum disorder (FASD)*

Recommendation

Strength of

Quality recommendationt of evidencet

\subsection{Screening, referral and support}

1.1 All pregnant and postpartum women should be screened for alcohol use with validated measurement tools by service providers who have received appropriate training in their use. ${ }^{10}$ Women at risk of heavy alcohol use should receive early, brief interventions (i.e., counselling and/or other services).

1.2 Referral of individuals for a possible FASD diagnosis should be made whenever there is evidence of, or suspected prenatal alcohol exposure at levels associated with, physical or developmental effects.

1.3 Abstinence from alcohol should be recommended to all women during pregnancy to ensure the safest outcome for the fetus, and appropriate support should be provided, as indicated.

Strong

High

\subsection{Medical assessment $\ddagger$}

2.1 The diagnostic process should include compiling a social and medical history and complete physical examination.

2.2 Confirmation of prenatal alcohol exposure requires documentation that the biological mother consumed alcohol during the index pregnancy based on: reliable clinical observation; self-report; reports by a reliable source; medical records documenting positive blood alcohol concentrations; alcohol treatment; or other social, legal or medical problems related to drinking during the pregnancy. The presence of all three facial features has such high specificity to alcohol exposure and FASD that confirmation of alcohol exposure is not required. ${ }^{11}$ The presence of fewer than three facial features does not have the same degree of specificity and therefore requires other confirmation.

\subsection{Sentinel facial features}

3.1 The following three sentinel facial features must be present because of their specificity to prenatal alcohol exposure:

- Palpebral fissure length $\geq 2$ SDs below the mean ( $<$ third percentile).

- Philtrum rated 4 or 5 on 5-point scale of the University of Washington Lip-Philtrum Guide. ${ }^{12}$

- Upper lip rated 4 or 5 on 5 -point scale of the University of Washington Lip-Philtrum Guide. ${ }^{12}$

\subsection{Neurodevelopmental assessment}

4.1 A diagnosis of FASD is made only when there is evidence of pervasive brain dysfunction, which is defined by severe impairment in three of more of the following neurodevelopmental domains: motor skills; neuroanatomy/neurophysiology; cognition; language; academic achievement; memory; attention; executive function, including impulse control and hyperactivity; affect regulation; and adaptive behaviour, social skills or social communication.

4.2 Severe impairment is defined as a global score or a major subdomain score on a standardized neurodevelopmental measure that is $\geq 2$ SDs below the mean, with appropriate allowance for test error. In some domains, large discrepancies among subdomain scores may be considered when a difference of this size occurs with a very low base rate in the population $(\leq 3 \%$ of the population). Clinical assessment with converging evidence from multiple sources and DSM-V diagnostic criteria ${ }^{13}$ for certain disorders may also be considered in specific domains that are not easily assessed by standardized tests. For example, in the affect regulation domain, the following diagnoses may be taken as an indication of severe impairment: major depressive disorder (with recurrent episodes), persistent depressive disorder, disruptive mood dysregulation disorder, separation anxiety disorder, selective mutism, social anxiety disorder, panic disorder, agoraphobia or generalized anxiety disorder). A domain-by-domain discussion of how these criteria are operationalized is outlined in Appendix 1 (available at www.cmaj.ca/lookup/suppl/doi:10.1503/ cmaj.141593/-/DC1).

\subsection{Nomenclature and diagnostic criteria}

5.1 A diagnosis of FASD may be made if an individual meets either of the two sets of criteria below: 5.1.1 FASD with sentinel facial features

- Simultaneous presentation of the three sentinel facial features (see section 3.0); AND

- Prenatal alcohol exposure confirmed or unknown; AND

- Evidence of impairment in three or more of the identified neurodevelopmental domains (see section 4.0) or, in infants and young children, evidence of microcephaly.

OR

5.1.2 FASD without sentinel facial features

- Evidence of impairment in three or more of the identified neurodevelopmental domains (see section 4.0); AND

- Confirmation of prenatal alcohol exposure, with the estimated dose at a level known to be associated with neurodevelopmental effects. 
Table 1 (part 2 of 2): Recommendations for the diagnosis of fetal alcohol spectrum disorder (FASD)*

Recommendation

5.2 At risk for neurodevelopmental disorder and FASD, associated with prenatal alcohol exposure

5.2.1 This is not a diagnosis; this is a designation that should be given to individuals when:

- There is confirmation of prenatal alcohol exposure, with the estimated dose at a level known to be associated with neurodevelopmental effects;

- Central nervous system criteria from 5.1.1 and 5.1.2 are not met;

- There is some indication of neurodevelopmental disorder in combination with a plausible explanation as to why the neurodevelopmental assessment results failed to meet the criteria for substantial impairment (e.g., patient was too young; incomplete assessment).

5.2.2 This designation may also be considered for individuals with all three sentinel facial features as described in 5.1.1 who do not yet have documentation or evidence of the requisite three or more neurodevelopmental domain criteria or true microcephaly. This designation should never be considered when prenatal alcohol exposure is confirmed absent.

\subsection{The diagnostic team}

6.1 Core team members across the lifespan:

For infants $(<18 \mathrm{mo})$

- Pediatrician/physician

- Child development specialist who has the skill set to conduct physical and functional assessments (e.g., speech-language pathologist, physiotherapist, occupational therapist, clinical psychologist)

For preschoolers (18 mo-5 yr)

- Pediatrician/physician

- Occupational therapist

- Speech-language pathologist

- Psychologist

For school-aged children (6 yr-age of majority)

- Pediatrician/physician with expertise in FASD and differential diagnosis

- Occupational therapist

- Speech-language pathologist

- Psychologist

For adults

- Physician

- Psychologist

- Speech-language pathologist/psychologist with expertise in language assessment

\subsection{Special considerations in neurodevelopmental assessment of infants and young children}

7.1 Infants and young children with all three sentinel facial features and microcephaly should be given a diagnosis of "FASD with sentinel facial features"; these children have a high risk of neurodevelopmental disorder. ${ }^{11,14}$ They should also be referred to a clinical geneticist.

7.2 Infants and young children with all three facial features may be given a diagnosis of "FASD with sentinel facial features" if they undergo a comprehensive neurodevelopmental assessment and show deficits in three or more brain domains. Infants and young children with confirmed prenatal alcohol exposure may be given a diagnosis of "FASD without sentinel facial features" if they undergo a comprehensive neurodevelopmental assessment and show deficits in three or more brain domains.

7.3 Infants and young children with confirmed prenatal alcohol exposure but who do not meet the criteria for FASD should be designated as "At risk for neurodevelopmental disorder and FASD, associated with prenatal alcohol exposure." Those with all three facial features but no microcephaly should be referred to clinical genetics.

7.4 A complete neurodevelopmental assessment should be recommended at an age-appropriate time for all infants and young children with confirmed prenatal alcohol exposure and/or all three facial features.

\subsection{Special considerations in neurodevelopmental assessment of adolescents and adults}

8.1 Recommendations following the assessment must address basic and immediate needs of the client, and assist them in accessing required resources.

\subsection{Management and follow-up}

9.1 Individuals with FASD and their caregivers should be linked to resources that can improve outcomes. However, just because availability of services is limited, an individual should not be denied an assessment and management plan. Often the diagnosis is the impetus that leads to the development of resources.

9.2 When young adults are transitioning to independent living situations, it may require that they undergo a reassessment to identify changes in their adaptive function and to make subsequent adjustments to their management plan.

Strong

High

Note: DSM-V = Diagnostic and Statistical Manual of Mental Disorders. Fifth Edition; SD = standard deviation.

*Appendix 1 provides details of the evidence underpinning these recommendations.

tUsing GRADE. ${ }^{7-9}$ See Box 1 for definitions.

fIncludes family history, maternal alcohol history, physical examination and differential diagnosis. 
length guideline in Appendix 1. An algorithm for diagnosis is available in Figure 1.

Clinicians must consider the issue of differential diagnosis when providing a diagnosis of FASD. Diagnosing FASD is often complex because of the presence of multiple risk factors and negative exposures that are substantial contributors to the patient's symptoms. No neurodevelopmental deficits are considered pathognomonic for, or specific to, FASD.

\section{Implementation}

Training programs are under development across Canada, as are presentations at national and international conferences to disseminate the revised and updated diagnostic guideline. Papers will be drafted focusing on the importance of FASD diagnosis and will be directed to health practitioners and the general public. The committee is also collaborating with professional societies (e.g., the Society of Obstetricians and Gynaecologists of Canada) to develop materials and assessment tools. The guideline will be re-evaluated when substantial new evidence emerges.

\section{Other guidelines}

Similar diagnostic approaches have been adopted internationally, but they differ in the specificity of recommendations, criteria and clinical cut-offs. ${ }^{13,15-19}$ Several important differences ex-

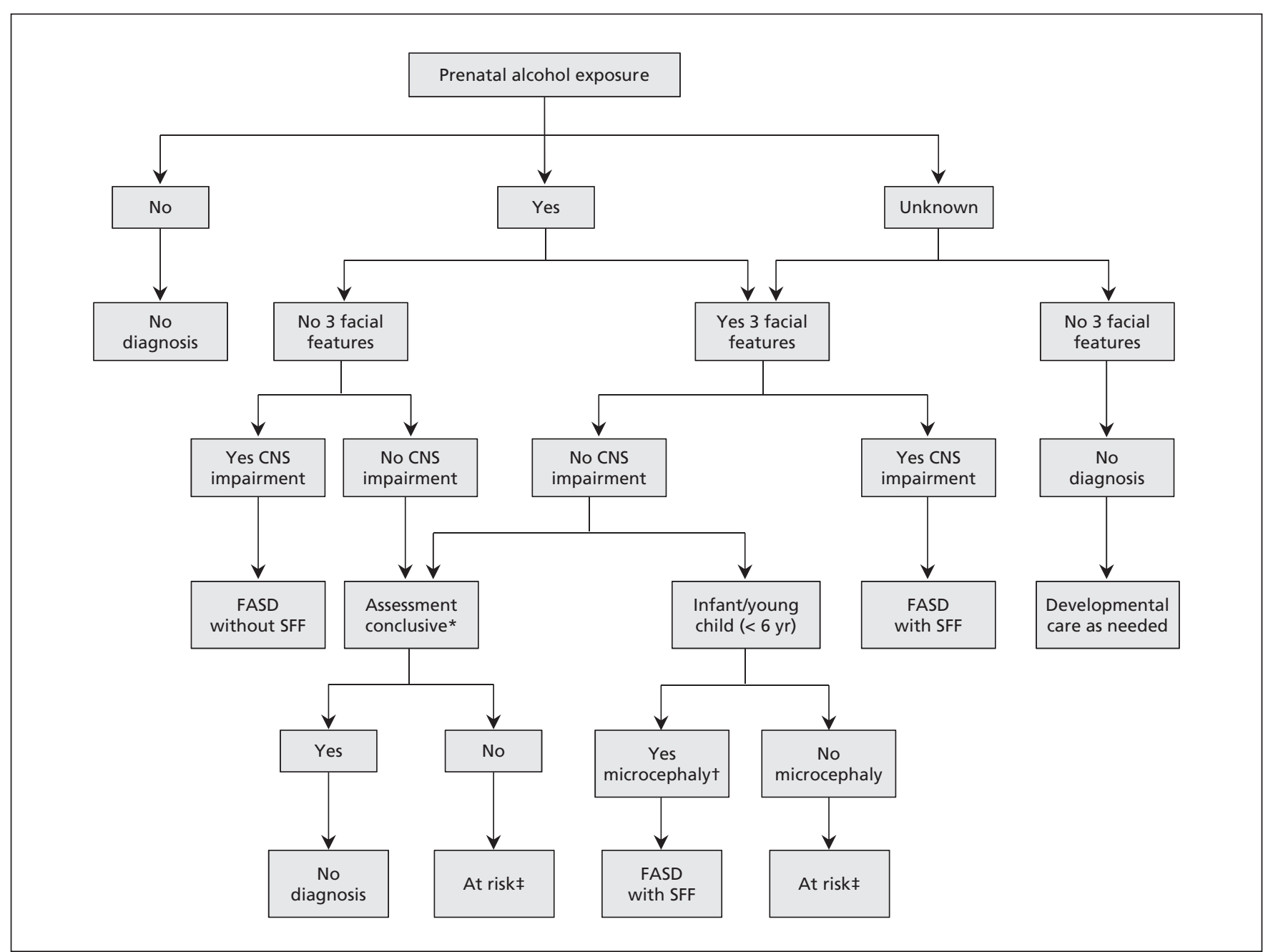

Figure 1: Diagnostic algorithm for fetal alcohol spectrum disorder (FASD). *Assessment conclusive = clinician conducting the neurodevelopmental assessment is satisfied that the session was a true representation of the person's ability and that any deficits reported were not due to extenuating circumstances. Assessments may be inconclusive for children under six years of age, because some domains cannot be assessed with confidence until the person is older or because of other confounding factors, such as temporary life stress or illness; see the text for more information. tMicrocephaly is not the only pathway to diagnosis for infants and young children; these individuals may also receive other FASD diagnoses, as specified elsewhere in the algorithm, if they show three areas of substantial impairment on neurodevelopmental tests. ¥At risk for neurodevelopmental disorder and FASD, associated with prenatal alcohol exposure. An at-risk designation includes situations where a full neurodevelopmental assessment is not conclusive because of age or situational factors; therefore, FASD may not be the diagnosis. Clinical judgment is recommended. Note: CNS = central nervous system (yes/no impairment in $\geq 3$ brain domains), SFF = sentinel facial features. 


\section{Box 2: Summary of specific updates in this guideline}

- The use of fetal alcohol spectrum disorder (FASD) as a diagnostic term

- The inclusion of special considerations for diagnosing FASD in infants, young children and adults

- The deletion of "growth" as a diagnostic criterion ${ }^{21}$

- The addition of a new "at-risk" category that will capture individuals who do not meet the diagnostic criteria but are still at risk of FASD

- The revision and refinement of brain domains evaluated in the neurodevelopmental assessment; specific changes and additions include:

- "Hard and soft neurological signs including sensory motor" was renamed "motor skills" and redefined

- "Brain structure" was renamed "neuroanatomy/neurophysiology" and redefined

- "Communication" was renamed "language"

"Attention deficit/hyperactivity" was renamed "Attention" and redefined

"Affect regulation" was added

"Executive function" was expanded and clarified

ist between this guideline and others; however, with the introduction of the new nomenclature, it is difficult to compare them directly. In general, diagnostic guidelines differ on the number of sentinel facial features required to make a diagnosis; the inclusion of growth deficits as a diagnostic criterion; the neurodevelopmental assessment process and criteria; and the approaches and measures used to confirm prenatal alcohol exposure (see Appendix 1 for further discussion). Recent data ${ }^{20}$ indicate that most Canadian clinics use the 2005 guideline. ${ }^{5}$ Box 2 outlines the differences between the 2005 guideline ${ }^{5}$ and this update.

\section{Gaps in knowledge}

Despite 40 years of research, there are still gaps in knowledge and evidence related to the diagnosis of FASD. For obvious ethical reasons, it is impossible to perform titrated dose-response studies of prenatal alcohol exposure and subsequent teratogenicity that would undoubtedly provide more definitive answers. Despite this apparent limitation, researchers and clinicians continue to study the effects of prenatal alcohol exposure using different models and methodologies, and evidence continues to emerge that improves the understanding and knowledge base of FASD. For example, diagnostic biomarkers are under investigation, as are additional tools to assess sensory processing and integration dysfunction, and sleep disorders in those with FASD. ${ }^{22}$ Further research is required to determine the association between prenatal alcohol exposure and other mental health problems. Databases containing diagnostic data need to be analyzed for correlations between sentinel facial features and patterns of neurodevelopmental def- icits. Research is ongoing, and their findings may reveal novel approaches that can improve available technologies for screening, diagnosis and management.

\section{Conclusion}

It has been well documented that technology and health care costs are rapidly increasing and that health care systems are re-evaluating existing programs to develop more cost-efficient and effective practices and models. We anticipate that these updated evidence-based recommendations for best practices in the diagnosis of FASD will improve the current process and will lead to more efficient and effective care for affected individuals across their lifespan. Although the assessment is meant to provide information about individuals' strengths as well as their challenges and aims to inform interventions, it is not solely for the purpose of diagnosis.

\section{References}

1. Lemoine P, Harousseau H, Borteyru JP, et al. Les enfants de parents alcoolique. Ouest Med 1968;21:476-82.

2. Jones KL, Smith DW, Ulleland CN, et al. Pattern of malformation in offspring of chronic alcoholic mothers. Lancet 1973;1:1267-71.

3. Population by year, by province and territory (number). Ottawa: Statistics Canada; 2014.

4. Clarren SK, Lutke J, Sherbuck M. The Canadian guidelines and the interdisciplinary clinical capacity of Canada to diagnose fetal alcohol spectrum disorder. J Popul Ther Clin Pharmacol 2011;18:e494-9.

5. Chudley AE, Conry J, Cook JL, et al. Fetal alcohol spectrum disorder: Canadian guidelines for diagnosis. CMAJ 2005;172 (Suppl 5):S1-21

6. Brouwers MC, Kho ME, Browman GP, et al. AGREE II: advancing guideline development, reporting and evaluation in health care. CMAJ 2010;182:E839-42.

7. Balshem H, Helfand M, Schunemann HJ, et al. GRADE guidelines: 3. Rating the quality of evidence. J Clin Epidemiol 2011; 64:401-6.

8. Andrews J, Guyatt G, Oxman AD, et al. GRADE guidelines: 14. Going from evidence to recommendations: the significance and presentation of recommendations. J Clin Epidemiol 2013; 66:719-25.

9. Andrews JC, Schunemann HJ, Oxman AD, et al. GRADE guidelines: 15 . Going from evidence to recommendation-determinants of a recommendation's direction and strength. J Clin Epidemiol 2013;66:726-35

10. Loock C, Conry J, Cook JL, et al. Identifying fetal alcohol spectrum disorder in primary care. CMAJ 2005;172:628-30.

11. Astley SJ. Validation of the fetal alcohol spectrum disorder (FASD) 4-Digit Diagnostic Code. J Popul Ther Clin Pharmacol 2013;20:e416-67.

12. Astley SJ, Clarren SK. Measuring the facial phenotype of individuals with prenatal alcohol exposure: correlations with brain dysfunction. Alcohol Alcohol 2001;36:147-59.

13. American Psychiatric Association. Diagnostic and statistical manual of mental disorders. Fifth edition. Arlington (VA): American Psychiatric Publishing; 2013.

14. Astley SJ. Profile of the first 1400 patients receiving diagnostic evaluations for fetal alcohol spectrum disorder at the Washington State Fetal Alcohol Syndrome Diagnostic \& Prevention Network. Can J Clin Pharmacol 2010;17:e132-64.

15. Stratton K, Howe C, Battaglia FC, editors. Fetal alcohol syndrome: diagnosis, epidemiology, prevention, and treatment. Washington (DC): National Academy Press; 1996.

16. Hoyme HE, May PA, Kalberg WO, et al. A practical clinical approach to diagnosis of fetal alcohol spectrum disorders: clarification of the 1996 institute of medicine criteria. Pediatrics 2005; 115:39-47. 
17. Astley SJ, Clarren SK. Diagnosing the full spectrum of fetal alcohol-exposed individuals: introducing the 4-Digit Diagnostic Code. Alcohol Alcohol 2000;35:400-10.

18. The ICD-10 classification of mental and behavioural disorders: clinical descriptions and diagnostic guidelines. Geneva: World Health Organization; 1992.

19. Fetal alcohol spectrum disorders: guidelines for referral and diagnosis. Atlanta: US Centers for Disease Control and Prevention; 2004.

20. Clarren SK, Halliwell CI, Werk C, et al. Using a common form for consistent collection and reporting of FASD data from across Canada: a feasibility study. J Popul Ther Clin Pharmacol 2015;22:e211-27.

21. O'Leary CM, Nassar N, Kurinczuk JJ, et al. The effect of maternal alcohol consumption on fetal growth and preterm birth. BJOG 2009;116:390-400.

22. Memo L, Gnoato E, Caminiti S, et al. Fetal alcohol spectrum disorders and fetal alcohol syndrome: the state of the art and new diagnostic tools. Early Hum Dev 2013;89(Suppl 1):S40-3.

Affiliations: Canada Fetal Alcohol Spectrum Disorder Research Network (Cook, Green, Lutke); Society of Obstetricians and Gynaecologists of Canada (Cook, Green), Ottawa, Ont.; Department of Obstetrics and Gynaecology (Cook), University of Ottawa, Ottawa, Ont.; Department of Biomedical and Molecular Sciences (Green), Queen's University, Kingston, Ont.; Sunny Hill Health Centre for Children (Lilley), Vancouver, BC; National Institutes of Health (Anderson), Ottawa, Ont.; Fetal Alcohol Spectrum Disorders Clinic (Baldwin), Child Development Services, Alberta Children's Hospital, Calgary, Alta.; Department of
Pediatrics (Chudley), University of Manitoba, Winnipeg, Man.; University of British Columbia (Conry [professor emerita]), Vancouver, BC; Department of Pediatrics (LeBlanc), Dr. Georges-L.-Dumont University Hospital Centre, Université de Moncton and Université de Sherbrooke, Moncton, NB; Department of Pediatrics (Looke), University of British Columbia, Vancouver, BC; Glenrose Rehabilitation Hospital (Mallon), Alberta Health Services, Edmonton, Alta.; Lakeland Centre for Fetal Alcohol Syndrome (McFarlane), Cold Lake, Alta.; Surrey Place Centre (Temple), Toronto, Ont.; Faculty of Medicine (Rosales), Memorial University of Newfoundland, St. John's, Nfld.

Contributors: All of the authors contributed substantially to the development of the guideline and to the drafting and critical revision of the manuscript. All of the authors gave final approval of the version to be published and agreed to act as guarantors of the work.

Funding: This project was funded by the Public Health Agency of Canada and the Canada Fetal Alcohol Spectrum Disorder Research Network.

Acknowledgements: The authors thank the many clinicians and individuals who helped develop, review and provide feedback on the guideline, especially Drs. Ilona AuttiRämö, Heather Carmichael Olson, Sterling Clarren, Claire Coles, Ana Hanlon-Dearman, Kim Kerns, Gideon Koren, Mansfield Mela, Mary O'Connor, Sarah Mattson and Edward Riley.

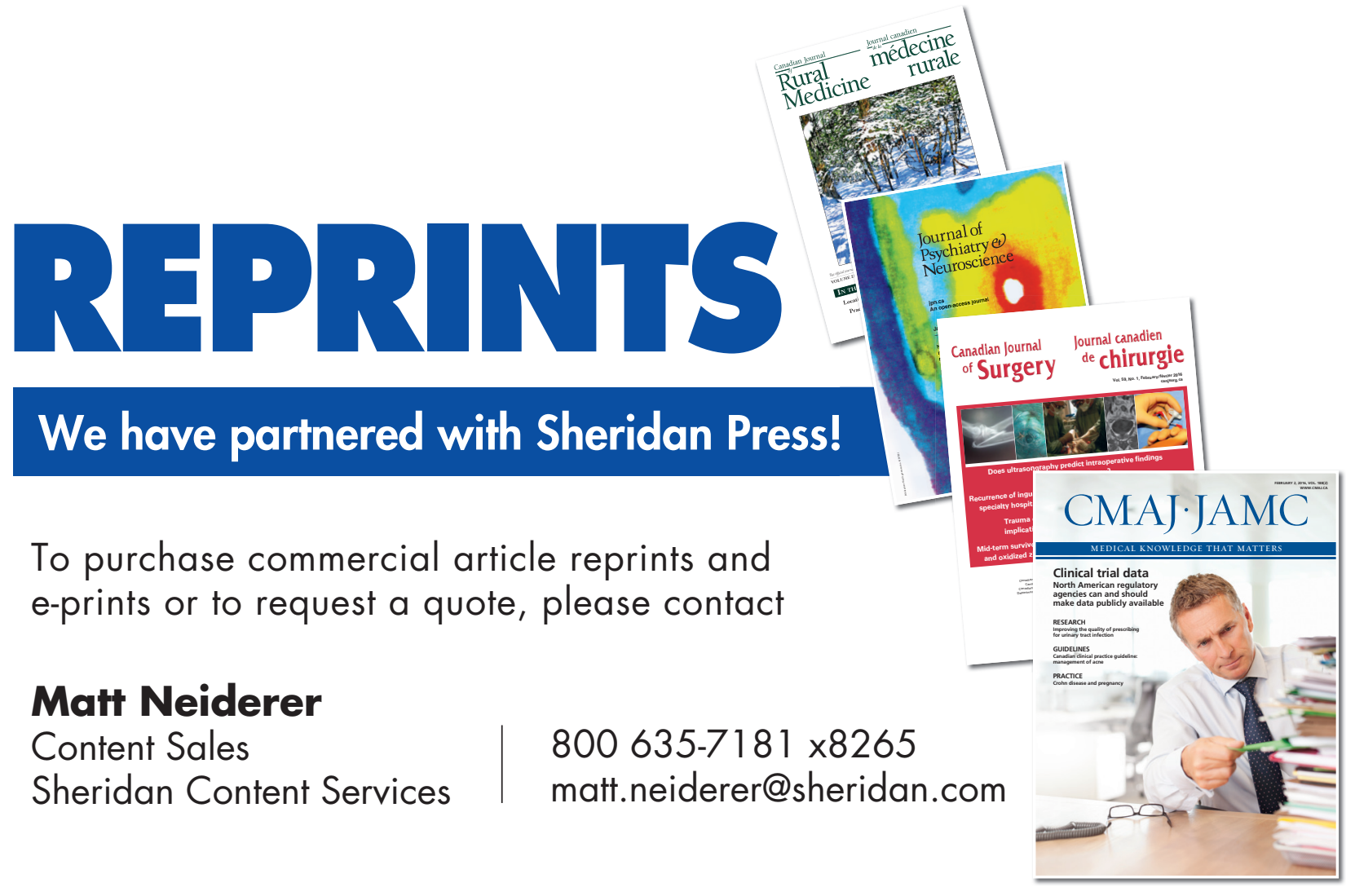

